\title{
Correction
}

\section{Correction to: Coupled Self-Organized Hydrodynamics and Stokes Models for Suspensions of Active Particles}

Pierre Degond®D, Sara Merino-Aceituno, Fabien Vergnet and Hui Yu

Correction to: J. Math. Fluid Mech. (2019) 21:6

$$
\text { https://doi.org/10.1007/s00021-019-0406-9 }
$$

This note provides a list of errata and their correction for Reference [1].

- There is a typo in Eq. (5.12): the term in $(\tilde{\lambda}-1)$ should come with a negative sign. This expression becomes:

$$
\begin{aligned}
& {\left[i \rho_{0}\left(-\alpha+V_{0} \cdot k\right)+\gamma|k|^{2} \rho_{0}-\frac{(\tilde{\lambda}-1) \tilde{b}}{2} \frac{\rho_{0}^{2} k_{0}^{2}}{|k|^{2}}\right] \bar{\Omega}} \\
& \quad=\left[\frac{1}{2} \frac{\rho_{0}}{|k|^{2}}\left(2 \tilde{\lambda} k_{0} \bar{p}+\tilde{b}(\tilde{\lambda}+1)\left(\rho_{0} \bar{k}+\bar{\rho} k_{0}\right)\right)-\frac{i}{\kappa} \bar{\rho}\right] k^{\perp} .
\end{aligned}
$$

- In the statement of Th. 5.1 (Linear Stability Analysis), we have that Eq. (5.5) is not true. As a consequence equation (5.6) is slightly modified into

$$
\begin{aligned}
D(\alpha, k)= & \left\{\frac { \tilde { b } \rho _ { 0 } } { 2 | k | ^ { 2 } } \left[\left(-4 \tilde{\lambda} \frac{k_{0}^{2}}{|k|^{2}}+\tilde{\lambda}+1\right)\left(-\alpha+U_{0} \cdot k\right)\right.\right. \\
& \left.\left.-c_{1} k_{0}\left(-2 \tilde{\lambda} \frac{k_{0}^{2}}{|k|^{2}}+\tilde{\lambda}+1\right)\right]+\frac{i}{\kappa} c_{1}\right\}\left(|k|^{2}-k_{0}^{2}\right) \\
& -\left(-\alpha+U_{0} \cdot k\right)\left[i\left(-\alpha+V_{0} \cdot k\right)-\frac{(\tilde{\lambda}-1) \tilde{b} \rho_{0}}{2} \frac{k_{0}^{2}}{|k|^{2}}+\gamma|k|^{2}\right],
\end{aligned}
$$

when $\bar{k} \neq 0$. And Eq. (5.7) is valid when $\bar{k} \neq 0$. When $\bar{k}=0$ we have a different scenario where $\bar{\rho}=0$ and $\bar{\Omega} \neq 0$ arbitrary and

$$
\alpha=V_{0} \cdot k+i\left(\frac{(\tilde{\lambda}-1) \tilde{b}}{2} \frac{\rho_{0} k_{0}^{2}}{|k|^{2}}-\gamma|k|^{2}\right),
$$

which gives only stable modes since $\tilde{\lambda} \in[-1,1]$ and so $\operatorname{Im}(\alpha) \leq 0$. (Finally, notice that, the parameter $\eta$ in the original article does not play a role any more in this corrected version.)

The original article can be found online at https://doi.org/10.1007/s00021-019-0406-9. 
To obtain these results we modify the proof of Th. 5.1 Case B, which we rewrite fully next (notice that the number of the equations that do not start by 0 refer to the number of the equations as they appear in the original article).

Proof. Case (B) Suppose that $k^{\perp} \neq 0$. Doing the inner product of Eq. (0.1) with $k$ and using that $k^{\perp} \cdot k=|k|^{2}-k_{0}^{2}$, the dispersion relation is given by (using Eq. (5.11)):

$$
\begin{aligned}
& \left\{\tilde{b} \frac{\rho_{0}}{2|k|^{2}}\left[\rho_{0} \bar{k}\left(-4 \tilde{\lambda} \frac{k_{0}^{2}}{|k|^{2}}+\tilde{\lambda}+1\right)+\bar{\rho} k_{0}\left(-\frac{2 \tilde{\lambda} k_{0}^{2}}{|k|^{2}}+\tilde{\lambda}+1\right)\right]-\frac{i}{\kappa} \bar{\rho}\right\}\left(|k|^{2}-k_{0}^{2}\right) \\
& \quad=\left[i \rho_{0}\left(-\alpha+V_{0} \cdot k\right)-(\tilde{\lambda}-1) \frac{\tilde{b} \rho_{0}^{2}}{2} \frac{k_{0}^{2}}{|k|^{2}}+\gamma|k|^{2} \rho_{0}\right] \bar{k},
\end{aligned}
$$

and from Eq. (5.10b) we have the relation

$$
\left(-\alpha+U_{0} \cdot k\right) \bar{\rho}+\rho_{0} c_{1} \bar{k}=0 .
$$

Next we distinguish between the cases $\bar{k} \neq 0$ and $\bar{k}=0$. Suppose that $\bar{k} \neq 0$, then from Eq. (0.5) we have that $-\alpha+U_{0} \cdot k \neq 0$ and multiplying Eq. (0.4) by $-\alpha+U_{0} \cdot k \neq 0$ and using Eq. (0.5), we get the dispersion relation in Eq. (0.2), after simplifying $\bar{k}$.

If, on the contrary, $\bar{k}=0$, then one can check that $k^{\perp} \cdot \bar{\Omega}=0$ (thanks to Eq. (5.10a)) and, therefore, they are normal. Since by assumption $k^{\perp} \neq 0$ from Eq. (0.1) we conclude that the coefficient in front of $k^{\perp}$ on the right hand side must be zero. Rewriting this term using that $\bar{k}=0$ and Eq. (5.11) we have that

$$
\bar{\rho} I=0,
$$

with

$$
I:=\tilde{b} \frac{\rho_{0}}{2|k|^{2}} k_{0}\left(-\frac{2 \tilde{\lambda} k_{0}^{2}}{|k|^{2}}+\tilde{\lambda}+1\right)-\frac{i}{\kappa} .
$$

From here we deduce that $\bar{\rho}=0$ because otherwise we should have that $I=0$ but the imaginary part of $I$ is non-zero, so $\bar{\rho}=0$. In particular this implies that Eq. (0.5) is fulfilled and that $\bar{\Omega} \neq 0$ (otherwise we would have null perturbation). Since $\bar{\Omega} \neq 0$, from Eq. (0.1) again (remembering that $k^{\perp} \perp \bar{\Omega}$ ) we must have that the coefficient in front of $\bar{\Omega}$ is equal to zero. This gives the dispersion relation (0.3).

Now, we go back to the case $\bar{k} \neq 0$. To simplify the analysis we will restrict ourselves to the case where $k^{\perp}=k$, i.e. $k_{0}=k \cdot \Omega_{0}=0$ and $\bar{k} \neq 0$. This implies, in particular, that $U_{0} \cdot k=V_{0} \cdot k=v_{0} \cdot k$. With these considerations one can simplify the dispersion relation (0.2) into

$$
\tilde{D}(\alpha, k)=0,
$$

where $\tilde{D}(\alpha, k)$ is given in Eq. (5.7).

- There are two typos at the end of page 21: in the third line of Case (A) part b) should read $\operatorname{Im}(\alpha)$ instead of $\operatorname{Im}(\omega)$; and in the fifth line should read " $\bar{\Omega}$ is arbitrary with $\bar{\Omega} \cdot \Omega_{0}=0$ " rather than " $\bar{\Omega}$ is arbitrary with $\bar{\Omega}, \Omega_{0} \neq 0 "$.

\section{Compliance with ethical standards}

Conflict of interest The authors declare they have no conflict of interest.

Open Access. This article is licensed under a Creative Commons Attribution 4.0 International License, which permits use, sharing, adaptation, distribution and reproduction in any medium or format, as long as you give appropriate credit to the original author(s) and the source, provide a link to the Creative Commons licence, and indicate if changes were made. The images or other third party material in this article are included in the article's Creative Commons licence, unless indicated otherwise in a credit line to the material. If material is not included in the article's Creative Commons licence and your 
intended use is not permitted by statutory regulation or exceeds the permitted use, you will need to obtain permission directly from the copyright holder. To view a copy of this licence, visit http://creativecommons.org/licenses/by/4.0/.

Publisher's Note Springer Nature remains neutral with regard to jurisdictional claims in published maps and institutional affiliations.

\section{Reference}

[1] Degond, P., Merino-Aceituno, S., Vergnet, F., Yu, H.: Coupled self-organized hydrodynamics and stokes models for suspensions of active particles. J. Math. Fluid Mech. 21, 6 (2019). https://doi.org/10.1007/s00021-019-0406-9

Pierre Degond and Sara Merino-Aceituno Department of Mathematics

Imperial College London

London SW7 2AZ

UK

e-mail: pdegond@imperial.ac.uk

Sara Merino-Aceituno

e-mail: s.merino-aceituno@sussex.ac.uk; sara.merino@univie.ac.at

Sara Merino-Aceituno

Faculty of Mathematics

University of Vienna

Oskar-Morgenstern-Platz 1

1090 Vienna

Austria

and

Department of Mathematics

University of Sussex

Falmer BrightonBN1 9RH

UK

Fabien Vergnet

Laboratoire de mathématiques d'Orsay (LMO), Université

Paris-Sud, CNRS

Universit Paris-Saclay

15 rue Georges Clémenceau

91405 Orsay Cedex

France

e-mail: fabien.vergnet@math.u-psud.fr

(published online: September 4, 2020)
Hui Yu

Institut für Geometrie und Praktische Mathematik

RWTH Aachen University

52062 Aachen

Germany

e-mail: huiyu@tsinghua.edu.cn

and

Mathematical Sciences Center

Tsinghua University

Haidian District Beijing100084

China 